\title{
E-learning and ICTs Applications in Nutrition Science
}

\author{
http://dx.doi.org/10.3991/ijes.v1i2.3279 \\ Athanasios Drigas, Maria Karyotaki \\ N.C.S.R. 'Demokritos', Institute of Informatics and Telecommunications, Telecoms Lab - Net Media Lab
}

\begin{abstract}
ICTs have become a strong ally in strengthening individuals' healthy lifestyle, taking into account nutrition intake and physical activity levels. Nutrition applications provide the means for automatic dietary intake and energy expenditure measurements as well as personalised counselling and educational services. Behaviour targeted applications should be appealing and intriguing, as they apply to many people with a variety of needs for a long period of time. Therefore, they should be efficient, accurate and functional. It is a fact that nutrition applications are by all means a cost-effective innovation by reducing the chasm between the individuals in need for constant interaction and their caregivers.
\end{abstract}

Index Terms - Icts technologies, nutrition applications, personalised nutrition.

\section{INTRODUCTION}

Contemporary nutrition-related issues in conjunction with innovations in information and communication technologies (ICTs) are the core-elements of the present stateof-the-art article. In an attempt to address to the societal needs for continuous health and nutrition preservance and care, ICTs provide the means to the realization of effective state policies and scientific interventions. Target audience of such applications is individuals at home or at work as well as professional caregivers. In both cases, the goal of the nutrition interventions implemented should be lifestyle-oriented. In order to fulfill these goals, applications should enhance end-users' self-monitoring and selfmanagement skills. ICTs are an inadversely strong determinant factor as to the massive, immediate and low-cost deployment of nutrition-related interventions.

Nutrition applications go far beyond the boundaries of mare consumption of low-calorie foods and one-way dietary interventions. They have to do with individuals' physical and psychological health as a unity. Therefore, manual, visual or audio dietary intake entry combined with activity level recordings or total energy expenditure measurements, should both be accompanied by counseling for raising self-awareness and self-efficacy levels. In essence, individuals are trained to change their behaviour by realizing their nutritional and lifestyle, less beneficiary habits. During the whole process, support and guidance are predictive factors of long-term engagement in a program. That is the reason why nutrition applications should adapt to individuals' personal profile and character for goalsetting as well as for intervention evaluation purposes. Tailored feedback towards individuals has been found to raise their accountability and offer them encouragement. Thus, the more personalized the feedback the more effective the intervention.
Effective applications should therefore be interactive in order to maintain individuals' interest as well as flexible and reusable in order to apply to as many individuals as possible at the minimum cost. Individuals' motivation is also enhanced through accessible, convenient and reliable applications, especially for multi-variable settings, such as life-long diseases and/or socially impaired target groups, who come up against a variety of everyday adversities. Professional carers are also in need of such applications as they seek to satisfy their constant need for efficient and high-quality counseling services. In general, nutrition applications provide quality and standardization of health intervention studies in an attempt to improve all people's nutritional and lifestyle behaviors.

The nutrition applications presented below, belong to the scientific field of "nutrition \& ICTs", which has been divided into six distinct parts according to their main use or special assignment.

\section{INFORMATICS/ARTIFICIAL INTELLIGENCE \& NUTRITION}

In this area, search is centred in the development of multi-level systems, designed to offer multi-purposed services for covering a variety of needs on behalf of their users.

Chena et al. launches a web-based nutrition diagnosis expert system for dietetic professionals in Taiwan. This expert system assists in the nutrition-related decision making by generating a patient's nutrition diagnosis, which is a three-faceted process. First of all, the system takes into consideration the nutritional conditions and problems associated with each patient. Second, an etiology of the patient's condition is described in order to set the goals for the nutritional care intervention. Finally, the patient's sighs and symptoms are recorded, including biochemical data, anthropometric measurements, physical examination findings and nutrition history. Therefore, the nutrition diagnosis expert system offers a holistic approach in nutrition diagnosis and management, as it comprises both identification of the problem and the means to deal with it in a standardized, profound and efficient way [1].

Lewis et al. argue that information technology may influence consumers' food choices by increasing their personal interest in looking after their own health status. Especially, hand-held devices or phones could record daily nutrient information during food intake and furthermore, a more personalized profile of the consumers' nutritional needs would be possible. Also, devices such as the bodybugg that measures the total energy expenditure of a whole day and sends it to an online database could pro- 
vide the interested party with the necessary feedback in order to achieve body weight goals [2].

Koch et al. bring to light an expanding, crossdisciplinary research subject concerning the delivery of care to older people, such as their nutrition, physical activity and medication with the assistance of information technology, sensor technology, and information systems. It seems that elderly population's perspectives towards their care differentiate according to the stage of their aging process as well as to their gender [3].

In Lopez et al., motion sensors inside a mobile phone convert accelerometry counts into energy expenditure by taking into account heart rate, body and environment temperature under free-living conditions. Such measurements may be also effective as far as the prevention of cardiovascular accidents is concerned [4].

Bickmorea et al. launch an ontology of health behaviour change, deployed for diet promotion. The notable efficacy of this automated, media-based intervention is attributed both to its capacity to emulate the behaviour of an expert counsellor and to its reusability and interoperability. In this way, patients' health behaviour change applications can become a strong health counselling tool, taking into account the reduction of their dissemination cost [5].

Takahashi et al. present an Automatic Nutrient Calculation System on the web, which identifies and calculates the amount of the ingredients in grams included in almost 1 million registered recipes as well as the total amount of nutrients in each one of the ingredients. In the ingredient nutrient database, information on the cooking processing method, change the quantity of nutrients [6].

Amft at al. argue that on-body sensors can make rough estimations on ratio of fluid and solid foods consumed and also they can capture food category and timing information. Thus, behavioral-based, nutrition interventions can become more solid and precise. The three sensing domains are arm and trunk movements, chewing of foods and swallowing activity. Evidently, recognition of dietary activity events was realized by means of quantitative detection and classification, accounting for automatic dietary monitoring [7].

Ayres et al. made a record on the necessary nutrition informatics competencies in dietetics. Informatics includes the efficiency to collect, store, retrieve and study data. Nutrition informatics is the intersection of information, nutrition, and technology, a future-holding, crossdisciplinary innovation in dietetics and health care in general. In Delphi study presented, registered dieticians are convinced of the fact that they should be able to select, implement and maintain sufficiently, information management systems across all levels of their practice [8].

\section{MOBILES \& NUTRITION}

Mobile nutrition systems offer the means for measuring food intake and energy expenditure as well as they provide constant communication and interaction in the form of personalised information exchange between the interested party and a professional adviser.

Hebden et al, describe the process of developing four smartphone applications to raise young adults' motivation in the improvement of their nutrition and physical activity behaviours. The purpose of the applications was to enhance subjects' self-reflection on their physical activity and consumption of take-out foods (fast food), fruit, vegetables and sugar-sweetened drinks. The realisations of such intriguing, cost-effective, long-term health interventions, focusing on subject's self-assessment and awareness, necessitate, therefore, continuous encouragement and support in the form of personalised feedback [9].

In Kerr et al., a 6-month nutrition intervention project aiming at young adults' improvement of eating behaviors, makes use of a mobile application called "CHAT" that keeps a record of the food images consumed as well as invites the intervention group to perform dietary changes through tailored feedback on their food intake via a text message. In this case, nutritional messages referred to consumption of fruit, vegetable and junk food are related to age, gender and behavioral characteristics. Not only are the content, frequency and length of text message important, but also the adequate time of receiving it in order for the participants to build their self-efficacy on healthy eating habits [10].

Vandelanotte et al. performed a pilot-study concerning middle-aged, Australian men views on mobile phone delivered physical activity and nutrition interventions. Middle-aged men prefer tailor-made, self-monitoring and intervention delivery applications performed especially in smartphones, with an emphasis on maximum simplicity, speed and convenience in their operation [11].

Medhi et al., present observations and findings from a three-month field study in rural India, during which an easy-to use application, deployed in low-cost mobile phones, substituted paper-based data for a more accurate and accessible, digital database concerning the combat against children's malnutrition. Data entry and management was upgraded, whereas infrastructure deficiencies should be diminished by staff training and easy-to access technical support [12].

\section{TELE-NUTRITION}

Applications belonging in telenutrition research field are easy-to-use and cost-effective, as they enhance the adaption of long-lasting, self-monitoring health behaviour change.

Hercberg et al. investigate the relationship between nutrition and health outcomes in a 10-year follow-up, webbased study called "The Nutrinet-Sante Study", located in France. At the starting point of the study, volunteers record three $24 \mathrm{~h}$ dietary intake, socio-demographic and lifestyle questionnaires, health, anthropometric questionnaires and physical activity questionnaires. Every year, the participants must once again fill in the abovementioned questionnaires as well as the three $24 \mathrm{~h}$ dietary records. Moreover, every month they will receive informative e-mails, reminding them of the necessity to update their personal profiles on their food behaviour, nutritional and health by filling out a new questionnaire via the website [13].

Neuenschwander et al. made a comparative study between a web-based and an in-person nutrition education program for low-income adults. The technical part of the interventions, such as their content and duration was similar. Traditional in-person nutrition education and webbased nutrition education, both showed significant nutrition-related behaviour outcomes. Moreover, the nutritionrelated changes were equivalent in both intervention groups. Web-based participants also reported willingness to use the website again, taking into account its efficacious 
design, decreased cost of accessibility and easy implementation [14].

Vandelanotte et al. made a pilot-study aimed to examine middle-aged, Australian men's opinions and perceptions regarding the use of internet in the improvement of their physical activity and nutrition behaviors. It is noteworthy that the aforementioned target group show low commitment levels to engagement in health intervention programs. Indeed, middle-aged men support the use of websites as a means to self-monitor their physical activity and nutrition behaviours on the condition that delivered interventions are accessible, understandable, appealing, reliable and concise [11].

Gibney et al. argue that the use of web-based, personalised nutrition applications for the collection of food data in an intervention study should be, first of all, plausible and user-friendly, even though precise and exact data entry from the participants is less possible. Furthermore, personalised feedback towards the participants should be easy-to-use, based on simple visual tools, instead of any numeric data. Food choice advice should be focused on meal intake and ranges of nutrient intake, presented on the computer screen by making a classification, depending on the participant's average nutritional needs [15].

Hong et al. created a kid-friendly, web-based nutrition education searching system, combining both video scripts of the cooking process of healthy recipes and easily learned nutrition information with plenty of searching methods. Children can seek for a menu of their preference by using a key word expression such as food materials, age group, menu type, menu style and nutrients or the upper and lower bound of the calories and the nutrients they opt for [16].

Hong et al. created a web expert system for nutrition management and counselling, which takes into consideration gender, age and diseases so as to compose general and therapeutic meals. The system compares e-databases, originating from user's information and experts' recommendations in the sense that the latest assess on-line the nutrients and calories included in a meal, chosen by the user of the system [17].

Vereecken et al. made a comparative study on the feasibility of young children's nutrition assessment, based on their dietary habits and their parents' sociodemographic variables, by implementing either an on-line assessment tool or a paper and pencil questionnaire. No significant differences were found in relation to nutrient and food group recordings, except from water. Parents that preferred to fill in the pencil food diary were younger and had a lower education level. From the parents that completed the on-line questionnaire, the majority indicated that it was user-friendly, attractive and clear [18].

\section{DISTANCE LEARNING \& NUTRITION}

Distance learning in nutrition is an effective way of providing high quality, standardized knowledge as well as a disease preventive technique.

Busstra et al. attempted to create an efficient educational tool for producers and compilers of food composition data as well as for food composition data users, such as dieticians, epidemiologists, nutritionists, food scientists and medical doctors. This interactive e-learning module focuses on its activating character and self-driven knowledge acquisition base in order to stabilize learners' competency in high quality food composition data production and use. In addition, learning process is structured in a way that macronutrient analysis training would become suitable for non-chemists, thus making the course more adaptable to learners' heterogeneous needs. The postgraduate students participating in the course thought very highly of the aforementioned, digital learning material on account of both its educational and instructional principles [19].

Charrondierea et al. describe a distance-learning application called "The Food Composition Study Guide", which centralises on self-based learning and evaluation techniques in the areas of food composition, management and use. Every distance-learning program, such as the Study Guide is remotely accessible by using electronic communication. Moreover, its design and delivery make it a well-plausible didactic tool to certify and broaden students' knowledge in a standardised and cost-effective way, improving the availability, comparability and quality of food composition data [20].

Steyn et al., made a research in the field of a postgraduate, distance course, taking place in South Africa, where nutritional needs of the people suffering from the Acquired Immune Deficiency Syndrome (AIDS) as part of the socio-economic impact of HIV and AIDS pandemics, was never investigated or described before. Postgraduate students' views on both the design and the implementation of the e-learning programme were rather positive, although it seemed unavoidable that their personal uncapabilities or constraints in computer literacy and internet access would lead to an undesirable failure in the fulfilment of the learning goals. Moreover, the students' positive learning experiences elevate as long as their personal needs and capabilities are taken into account, including any shortages in the necessary resources such as accessible technology, budget and available time [21].

Martínez et al., created a transdisciplinary, collaborative, postgraduate project, powered by wikispaces, concerning the Mediterranean Diet and its social, economic, cultural and health related facets. According to students' evaluation of the project, the holistic and collaborative learning approach about the Mediterranean Diet was followed by the acquisition of additional competences and skills, such as the effective use of information and communication technology [22].

Trovato et al., suggest that professional post-graduate training in experts' enhanced skills and knowledge for convincing patient's to adapt to the Mediterranean Diet lifestyle, through e-learning, is a fundamental tool for the fulfilment of "Predictive, Preventive and Personalised Medicine". Predictive Medicine is based upon the clinical excellence of younger doctors in relation to their subject's nutrition and physical activity assessment. Preventive Medicine counters with the establishment of nutritionfriendly schools, striving for children's balanced diet. Finally, Personalised Medicine binds the subject's tailored and timely diagnosis with nutrition and lifestyle changes. Overall, dieticians and medical doctors must share skills and competences in order to boost their patient's motivation and self-efficacy, making clear of the fact that behavioural psychology must have a vital role in Continuous Medical Education [23].

Tao made an in-depth research in Taiwanese college students' perceptions over e-learning and online asyn- 
chronous education programs in general. Issues of interest were students' performance, student satisfaction and dropout rate. Discernible typology of different student groups came up from data analysis. Thus, the sceptics and the optimists emerged from a factor analysis of 30 variables. The results of the study may assist stakeholders' elearning decisions and furthermore promote e-learning programs [24].

Rudolf et al. designed an intervention study called "HENRY", Health Exercise Nutrition for the Really Young, aiming to help practitioners work more effectively on obesity prevention and treatment, with parents whose children are under the age of five. The intervention involved face-to-face group training and an e-learning course, both structured in a self-reflective way to boost practitioners' confidence on addressing to parents' taking up on healthier lifestyle. The participants' self-assessment as well as their managers' post-training evaluation showed that the program helped first of all the practitioners and their families develop better eating behaviours and therefore, enhanced their nutrition and emotional wellbeing as a whole. Indeed, the program helped the participants and the parents collaborate better through common decision making and by working in a more supportive climate [25].

\section{OBESITY \& NUTRITION}

Obesity targeted applications have to do both with weight-loss intervention programs as well as obesity prevention through the adoption of healthy lifestyle.

Mc Donald et al. made a survey, investigating pediatricians' familiarity with the use of Health Information Technology and more specifically with the Expert Committee Recommendations program for obesity screening and management. Researchers aimed to make an in-depth investigation around the pediatricians' perceptions in the use of electronic health records and decision support systems for the improvement of their nutrition and physical activity counselling. Findings depict clinicians' intention to stay focused on each patient's individual needs as well as to their own clinical experience when it comes to obesity management and counselling. However, they are in favor of implementing functional interventions, such as a decision alert that identifies obesity and is linked to targeted educational materials related to patient's age, gender, neighborhood and weight status [26].

Taylor et al. outline the outcomes from nutrition and physical activity interventions, centred in adult males via web-based mode of delivery. Particularly, studies aimed at showing a significant change in adult males' dietary intake or in their body weight and Body Mass Index over the intervention period. As far as the results of the studies are concerned, effectiveness of the internet-based features of the intervention delivery was insignificant due to the heterogeneity of the study designs. Nonetheless, adult men seem to be more easily recruited in a study if they can use the internet from their worksite environment. Moreover, instant and tailored feedback, such as clear, concise and achievable in goal setting messages presented in a comical manner, in response to adult men's self-monitoring of lifestyle changes, are strongly associated with the enhancement of the effectiveness of dietary interventions for males [27].

Nakandala et al. present a fuzzy logic methodology as to the determination of individual's carbohydrate intake requirements by integrating three input variables, level of physical activity, age and Body Mass Index. This multiple decision criteria application owns its fuzzy logic principles to experts' knowledge and its final goal is to enhance individual health sustenance in contemporary life conditions [28].

Storey et al. studied Canadian adolescents' current bodyweight status, nutrient intakes, meal behaviours and physical activity levels by using a web-based, selfadministered survey. According to the findings, overweight and obese students consumed significantly more total fat and high calorie beverages as well as higher frequency of breakfast skipping. It is crucial for adolescents' weight status to improve their lifestyle behaviours, including school health policies [29].

Lewis et al. note the importance of incorporating personal data such as Body Mass Index, activity level and individual dietary goals to provide tailored-made, health and nutrition intervention programs, which can be optimized by using a web-based interactive, self-monitoring mechanism. In concrete, effective intervention programs own their success, partly to participants' knowing exactly what they have to do to achieve their health and nutrition goals and having to be accountable for their own success. Continuous feedback, weekly or less frequently, rather aspiring for the participants, is indispensable [2].

Baños et al. describe an e-health platform for the treatment and prevention of childhood obesity, divided into three real-time, interconnected applications. The Clinical support system, the Home support system and the Mobile support system. Clinical support system is implemented by clinicians in order to create patient's medical and psychological profile through self-responsive questionnaires and furthermore, proceed to necessary treatment adaptations. Home support system provides children's selfaccess to educational games in an attempt to combine learning goals and amusement as well as to improve motivation levels and self-reinforcement of healthy nutrition and lifestyle behaviours. Finally, Mobile support system encompasses a Personal Digital Assistant, which operates on self-report bases for recording food intake and physical activity as well as a Sensorisation platform, which reserves students' physiological information and mobility habits. Generally speaking, self-report techniques are considered a reliable predictor of success during weight-loss treatments [30].

Shehu et al. found that Nigerian High school girls' percentage of body fat and activity pattern are related significantly. They also make clear of the fact that obesity can be combated only by both dietary restriction and increased physical exercise. Health education programs should raise children's interest on adopting healthy lifestyle to avoid getting fat [31].

Shaban-Nejad et al. have generated a consistent, multidisciplinary semantic platform that assembles social, environmental, economical and behavioural knowledge in the domain of healthy eating and child obesity prevention. Such biomedical ontologies, like the Childhood Obesity Prevention [Knowledge] Enterprise (COPE), include advanced logical reasoning services and social networking in order to develop an integrated consensus knowledge base. COPE can offer dietary recommendations based on the individual's personal profile for promoting healthy eating habits [32]. 
Dour et al. performed a web-based intervention study on weight-related health behaviour changes occurring in college students. Positive effects were found on fruit and vegetable consumption and maintenance of baseline levels of physical activity. Furthermore, motivation, program satisfaction, and achievement of intervention goals differentiate on account of the gender. Men's motivation originated from enhancing their personal goal-setting skills in contrary to their female counterparts, who found more motivating, the combination of health and pleasure [33].

Chen et al. attempted to promote Chinese American adolescents' skills in adapting to healthier lifestyle and body weight, by implementing a web-based intervention program that focused on participants' problem-solving skills and the setting of realistic and achievable personal goals as far as healthy food intake and active lifestyle is concerned. Results showed a reduction in waist-to-hip ratio and diastolic blood pressure, whereas there was an increase in vegetable and fruit intake as well as in levels of physical activity. In this program, adolescents' parents were guided via web-based sessions on the ways to improve their children's healthy weight-related behaviors, considering the high rate of Chinese parents' involvement in their children's lives [34].

Rusin et al. resume research data on informatics, food intake and nutrition. The target group of these applications is mainly overweight, obese and diabetes' patients. Most applications permit food type entry and quantity consumed by the individual, whereas others record carbohydrate or calorie intake. Meanwhile, mobile phones first and foremost, personal computers and personal digital assistants are the devices, used for personalized reports and data sharing. In order to simplify the food recording process and save time, search in a food database, reports, graphical presentations, listing of favourite foods and an overview of the user's own meals are optimized [35].

\section{DIABETES \& NUTRITION}

Diabetes-related applications attempt to address to diabetic patients' multiple needs both as far as self- implemented screening and management of the disease is concerned.

Bakken et al. made a research on urban and rural, older diabetic patients' satisfaction and usefulness over the implementation of Informatics for Diabetes Education and Telemedicine (IDEATel) project. Home telemedicine unit consisted of a video camera and microphone, a home glucose monitoring device and blood pressure cuff as well as access both to their clinical data profile and to an educational web site in low literacy version of English and Spanish. Results showed participants' perceptions of satisfaction and usefulness to be highly positive. Patients' location of residence (urban-rural) had little to do with their perceptions on utilisation, usefulness and satisfaction of the project. Thus, any associations found were attributed to distinct survey methods as data from urban participants were collected in a face-to-face interview rather than via mailed means, such as in case of the rural sample. Hispanic and non-Hispanic participants were treated equally in regards to materials of research and communication means as everything was available also in Spanish, except from English [36].

Spanakis et al. focus on the implementation of a novel care model for diagnosing and monitoring diabetes. The
Reaction project took place in Greece, Italy, Cyprus, France and participants noted that information technology may bring profound changes in patient's self-care and empowerment as well as in-depth communication between the latest and their health providers. Epatch technology, a small body-sensor adhesive to patient's skin, was used for measuring physiological signals and continuous glucose monitoring. Moreover, a platform provides professional decision support for in-hospital environments and safety monitoring for dosage and compliance, by implementing wireless technologies for continuous blood glucose control, clinical monitoring and intervention strategies, all in a closed-loop system. The Glucose Management System application, developed within the Reaction project, collects information from various sources such as glucose level, nutritional intake, administered drugs and the patient's insulin sensitivity so as to calculate the required insulin dosing during hospitalisation. Furthermore, insulin therapy should not be associated only with frequent blood glucose measurement, but also with controlling lifestyle factors to improve diabetics' overall health status [37].

$\mathrm{Yu}$ et al. present a support vector machine model that selects the most adequate sets of individuals' variables in order to achieve the best possible classification into common diseases like diabetes. More specifically, the set of diabetes-related variables was family history, age, race, ethnicity, weight, height, waist circumference, Body Mass Index, hypertension, gender and physical activity. The model evaluation showed that the support vector machine was an effective web-based tool, useful for the prediction of other common diseases, as well. Its main beneficiary characteristics are related to multivariate risk factors with small effects and unknown underlying biological relationships, let alone limited sample size [38].

Kaufman reports that computerised interventions empower patient's self-management of diabetes, lead to beneficiary behaviour changes and increase their knowledge through distance learning programs. To be more specific, effective interventions using information technology should be flexible, as they should address to every patient's individual goals and characteristics in order to maintain patient's interest over a long period of time. Patients' positive experiences with telemedicine interventions relate to their interaction with their clinical team or friends, besides being able to self-monitor glucose levels. In addition, improved clinical outcomes are accompanied by cell-phone and text message interventions, which have been found to increase patient-provider and parent-child communication as well as satisfaction with care. Finally, email-based behaviour change interventions have been found to increase physical activity and improve nutrition intake [39].

Sieverdes et al. made a review study on the guidelines and the use of mobile health technology as a means to prompt individuals' skills on self-management of diabetes and furthermore its care and health preservation. Glucose monitoring, medication dosage, nutrition therapy and blood pressure automatic feedback, strongly depend on the constant interaction between the patient and his/her health care providers in order to make any necessary adjustments in the implementation of the treatment. As far as nutrition therapy in diabetes is concerned, except from telemedicine practices that should incorporate voice recognition and visual feedback for recording food intake in food diary programs, it is vital for patients to be educat- 
ed on food selection, preparation and portion sizes. Successful adherence to physical activity is related to developing self-regulatory skills, for constant self-improvement and new goal setting. Internal accelerometers in smartphones, is a rather cost-effective application for measuring or tracking physical activity. Moreover, selfmonitoring of weight and body composition may increase patient's accountability, especially if their bodyweight changes are also monitored by peers via social networking sites. Overall, real-time messaging via mobile streaming services are used for patients' encouragement as well as for educatory and self-regulatory reasons, including algorithms that determine the amount of insulin or carbohydrates needed to regulate glucose. Future application design and messaging should focus more on the target audience, either through their intriguing and motivating characteristics or by leaving individuals some freedom of choice [40].

\section{CONCLUSION}

Research data on nutrition \& ICTs show the importance of behaviour-based applications in individuals' health preservance. Dietary intake and physical activity in everyday life are both constituents of a healthy lifestyle. Selfregulatory skills are vital for life-long behavioural changes. Nutrition applications should also be genuily intriguing as they need to provoke individuals' long-term engagement in an intervention program. For that purpose, personalised communication as well as tailored feedback according to individuals' personal profile, are predominant for treatment alterations and psychological support. Moreover, flexible, multiple purpose and cost-effective nutrition applications offer the opportunity of serving the needs of a large number of the population at a low cost, thus enhancing preventive medicine and reducing the inherent difficulties of the deprived part of the society. Finally, such applications should be accurate and trustworthy in order to gain interested party's acknowledgement and acceptance, ranging from healthcare professionals to individuals, concerned about their health status.

\section{REFERENCES}

[1] Chena Y., Hsua C.-Y., Liua, L., Yangb S. : "Constructing a nutrition diagnosis expert system", Expert Systems with Applications, vol. 39, issue 2, pp. 2132-2156, (2012). http://dx.doi.org/10.1016/j.eswa.2011.07.069

[2] Lewis K. D. \& Burton-Freeman B. M. : "The Role of Innovation and Technology in Meeting Individual Nutritional Needs", The Journal of Nutrition, vol. 140, pp. 426-436, (2010). http://dx.doi.org/10.3945/jn.109.114710

[3] Koch S. \& Hägglund M.: "Review: Health informatics and the

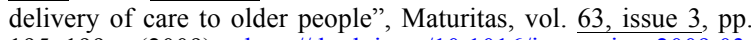
195-199, (2009). http://dx.doi.org/10.1016/j.maturitas.2009.03. $\underline{023}$

[4] Lopez L. J. R., Goroso D. G. and Battistella L. M. : "Sensor Network for Assessment of Energy Expenditure design based on Android CLAIB 2011", IFMBE Proceedings, vol. 33, pp.678-681, 201, (2011).

[5] Bickmorea T. W., Schulmana D. \& Sidnerb C. L : “A reusable framework for health counseling dialogue systems based on a behavioral medicine ontology", Journal of Biomedical Informatics, vol. 44, issue 2, pp. 183-197, (2011). http://dx.doi.org/10.1016/ j.jbi.2010.12.006

[6] Takahashi J., Ueda T., Nishikawa C., Ito T. \& Nagai A.: "Implementation of Automatic Nutrient Calculation System for Cooking Recipes Based on Text Analysis", PRICAI 2012, LNAI 7458, 789-794, (2012).
[7] Amft O. \& Tröster G. : "Recognition of dietary activity events using on-body sensors" Artificial Intelligence in Medicine, vol. 42, issue 2, pp. 121-136, (2008). http://dx.doi.org/10.1016/ j.artmed.2007.11.007

[8] Ayres E. J., Greer-Carney J. L., McShane, F. P. E., Miller, A. \& Turner P. : "Nutrition Informatics Competencies across All Levels of Practice: A National Delphi Study", Journal of the Academy of Nutrition and Dietetics, vol. 112, issue 12, pp. 2042-2053, (2012). http://dx.doi.org/10.1016/j.jand.2012.09.025

[9] Hebden L., Amelia Cook A., Van der Ploeg H. P. \& Margaret Allman-Farinelli M. : "Development of Smartphone Applications for Nutrition and Physical Activity Behavior Change", JMIR Res Protoc 2012, vol. 1, issue 2, e9, (2012).

[10] Kerr D. A., Pollard C. M., Howat P., Delp E. J., Pickering M., Kerr K. R., Dhaliwal S. S., Pratt L. S., Wright J. \& Boushey C. J. : "Connecting Health and Technology (CHAT): protocol of a randomized controlled trial to improve nutrition behaviours using mobile devices and tailored text messaging in young adults", BMC Public Health, vol. 12, issue 477, pp. 1-10, (2012).

[11] Vandelanotte C. , Caperchione C. M., Ellison M., George E. S., Maeder A., Kolt G. S., Duncan M. J., Karunanithi M., Noakes M. , Hooker C., Viljoen P. \& Mummery W. K. : "What Kinds of Website and Mobile Phone-Delivered Physical Activity and Nutrition Interventions Do Middle-Aged Men Want?", Journal of Health Communication, pp. 1-14, (2013).

[12] Medhi I, Jain M., Tewari A., Bhavsar M., Matheke-Fischer M. \& Cutrell E. : "Combating Rural Child Malnutrition through Inexpensive Mobile Phones", NordiCHI'12, October 14-17, 2012 Copenhagen, Denmark.

[13] Hercberg S., Castetbon K. Czernichow S., Malon A. Mejean C., Kesse E., Touvier M. \& Galan P. : "The Nutrinet-Sante Study: a web-based prospective study on the relationship between nutrition and health and determinants of dietary patterns and nutritional status", BMC Public Health, vol. 10, issue 242, pp. 1-6, (2010).

[14] Neuenschwander, Author VitaeL. M., Abbott, A. \& Author VitaeMobley, A. R. : "Comparison of a Web-Based vs In-Person Nutrition Education Program for Low-Income Adults", Journal of the Academy of Nutrition and Dietetics, vol. 113, issue 1, pp. 120126, (2013).

[15] Gibney M. J and Walsh M. C. : "The future direction of personalised nutrition: my diet, my phenotype, my genes", Proceedings of the Nutrition Society, vol. 72, pp. 219-225, (2013). http://dx.doi.org/10.1017/S0029665112003436

[16] Hong S.-M., Lee T.-K., Chung H.-J., Park H.-K., Lee E.-J., Nam H.-S., Jung S.-I., Cho J.-Y., Lee J.-H., Kim G. \& Kim M.-C. : "Construction of web-based nutrition education contents and searching engine for usage of healthy menu of children", Nutrition Research and Practice, vol. 2, issue 2, pp. 114-120, (2008). http://dx.doi.org/10.4162/nrp.2008.2.2.114

[17] Hong S.-M., Cho J.-Y., Lee J.-H., Kim G. \& \& Kim M.-C. : "NutriSonic web expert system for meal management and nutrition counselling with nutrient time-series analysis, e-food exchange and easy data transition", Nutrition Research and Practice, vol. 2, issue 2, pp. 121-129, (2008). http://dx.doi.org/10.4162/nrp. 2008.2.2.121

[18] Vereecken C. A., Covents M., Haynie D. \& Maes L. : "Feasibility of Young Children's Nutrition Assessment on the Web", J Am Diet Assoc, vol. 109, issue 11, pp. 1896-1902, (2009). http://dx.doi.org/10.1016/j.jada.2009.08.013

[19] Busstraa M. C., Hulshofa Paul J. M., Houwenb Jan, Elburga L., Peter C.H. \& Hollmana P. C. H. : "Nutrient analysis explained for non-chemists by using interactive e-learning material", Journal of Food Composition and Analysis, vol. 25, issue 1, pp. 88-95, (2012). http://dx.doi.org/10.1016/j.jfca.2011.07.003

[20] Charrondierea, R. U., Bermana S. \& Elmadfab I. : "Food composition training: Distance learning as a new approach and comparison to courses in the classroom", Journal of Food Composition and Analysis, vol. 22, issue 5, pp. 421-432, (2009). http://dx.doi.org/10.1016/j.jfca.2009.05.001

[21] Steyn L., Cronje J. C. \& Bothma T. J. D. : "E-learning: a nutrition and HIV/AIDS information tool", Perspectives, vol. 60, issue 4, pp. 364-382, (2008).

[22] Martínez A. A., Medina F. Xavier, Pons Albalat J. A. \& Rubio F. S. : "Desafíos y oportunidades de las herramientas 2.0 para el stu- 
PAPER

\section{E-LEARNING AND ICTS APPLICATIONS IN NUTRITION SCIENCE}

dio interdisciplinar de la nutrición: el caso de la Wiki Dieta Mediterránea", Revista de Universidad y Sociedad del Conocimiento (RUSC), vol. 10, issue 1, pp. 29-44, (2013).

[23] Trovato Guglielmo M. : "Behavior, nutrition and lifestyle in a comprehensive health and disease paradigm: skills and knowledge for a predictive, preventive and personalized medicine", Trovato EPMA Journal 2012, vol. 3, issue 8, (2012).

[24] Tao Yu-Hui : "Typology of college student perception on institutional e-learning issues - An extension study of a teacher's typology in Taiwan", Computers \& Education, vol. 50, issue 4, pp. 1495-1508, (2008).

[25] Rudolf, M. C. J., Hunt, C., George, J., Hajibagheri K. \& Blair M. "HENRY: development, pilot and long-term evaluation of a programme to help practitioners work more effectively with parents of babies and pre-school children to prevent childhood obesity", Child: care, health and development, vol. 36 , issue 6, pp. 850-857, (2010). http://dx.doi.org/10.1111/j.1365-2214.2010.01116.x

[26] McDonald J., Goldman R. E., O’Brien A., Ayash C., Mitchell K., Marshall R., Simon R. S. \& Taveras, E. M. : "Health Information Technology to Guide Pediatric Obesity Management", Clinical Paediatrics, vol. 50, issue 6, pp. 543-549, (2011). http://dx.doi.org/10.1177/0009922810395131

[27] Taylor P. J., Kolt G. S., Vandelanotte C., Caperchione C. M., Mummery W. K., George E. S., Karunanithi M. \& Noakes M. J. "A review of the nature and effectiveness of nutrition interventions in adult males - a guide for intervention strategies", International Journal of Behavioral Nutrition and Physical Activity, vol. 10 , issue 13, pp. 1-13, (2013).

[28] Nakandala D. \& Lau H.C.W. : "A novel approach to determining change of caloric intake requirement based on fuzzy logic methodology, Knowledge-Based Systems, vol. 36, pp. 51-58, (2012). http://dx.doi.org/10.1016/j.knosys.2012.05.020

[29] Storey K. E., Forbes L. E., Fraser S. N., Spence J. C., Plotnikoff R. C., Raine K. D. \& Mc Cargar L. J. : "Adolescent Weight Status and Related Behavioral Factors: Web Survey of Physical Activity and Nutrition", Journal of Obesity, pp. 1-8, (2012). http://dx.doi.org/10.1155/2012/342386

[30] Baños R. M., Cebolla A., Botella C., García-Palacios A., Oliver E., Zaragoza I. \& Alcaniz M. : "Improving Childhood Obesity Treatment Using New Technologies: The ETIOBE System", Clinical Practice \& Epidemiology in Mental Health, vol. 7, pp. 62-66, (2011). http://dx.doi.org/10.2174/1745017901107010062

[31] Shehu R. A., Onasanya S.A., Oloyede T. A. \& Kinta M. : "Contribution of Information and Communication Technology to the Prevalence of Obesity and Elevated Blood Pressure among Secondary School Students in Nigeria", Journal of Applied Sciences, vol. 10, issue 4, pp. 359-362, (2010).

[32] Shaban-Nejad, A. Buckeridge D. L., Dubé L. : “ COPE: Childhood Obesity Prevention [Knowledge] Enterprise”, AIME 2011, LNAI 6747, 225-229, (2011).
[33] Dour C. A., Horacek T. M., Schembre S. M., Lohse B., Hoerr S. Kattelmann K., White A. A., Shoff S., Phillips B., Greene G. : "Process Evaluation of Project WebHealth: A Nondieting Webbased Intervention for Obesity Prevention in College Students", Journal of Nutrition Education and Behavior, (2013). http://dx.doi.org/10.1016/j.jneb.2012.10.001

[34] Chen J.-L., Weiss S., Heyman M. B, Cooper B. \& Lustig R. H. : "The Efficacy of the Web-Based Childhood Obesity Prevention Program in Chinese American Adolescents (Web ABC Study)", J. Adolesc Health, vol. 49, issue 2, pp. 148-154, (2011). http://dx.doi.org/10.1016/j.jadohealth.2010.11.243

[35] Rusin M., Årsand E. \& Hartvigsen G. : "Functionalities and input methods for recording food intake: A systematic review", International Journal of Medical Informatics, (2013). http://dx.doi.org/10.1016/j.ijmedinf.2013.01.007

[36] Bakken S., Grullon-Figueroa L., Izquierdo R., Lee N.-J., Morin P., Palmas W., Teresi J., Weinstock R. S., Shea S., Starren J. : "Development, Validation, and Use of English and Spanish Versions of the Telemedicine Satisfaction and Usefulness Questionnaire", J Am Med Inform Assoc, vol.13, pp. 660-667, (2006). http://dx.doi.org/10.1197/jamia.M2146

[37] Spanakis E. G., Chiarugi F., Kouroubali A., Spat S., Beck P., Asanin S., Rosengren P., Gergely T., Thestrup J. : “ Diabetes Management Using Modern Information and Communication Technologies and New Care Models", Interact J Med Re, vol. 1, issue 2, e8, (2012). http://dx.doi.org/10.2196/ijmr.2193

[38] Yu, W., Liu T., Valdez R., Gwinn M. \& Khoury M. J. : “Application of support vector machine modeling for prediction of common diseases: the case of diabetes and pre-diabetes", BMC Medical Informatics and Decision Making, vol. 10, issue 16, e7, (2010).

[39] Kaufman N. : "Internet and information technology use in treatment of diabetes", Int J Clin Pract, vol. 64 (Suppl. 166), pp. 4146, (2010). http://dx.doi.org/10.1111/j.1742-1241.2009.02277.x

[40] Sieverdes, J. C., Treiber, F. \& Jenkins, C. : "Improving diabetes management with mobile health technology", The American Journal of the Medical Sciences, vol. 345, issue 4, pp. 289-295, (2013). http://dx.doi.org/10.1097/MAJ.0b013e3182896cee

\section{AUTHORS}

A. Drigas is with N.C.S.R. 'Demokritos', Institute of Informatics and Telecommunications, Telecoms Lab - Net Media Lab, Agia Paraskevi, 153 10, Athens, Greece ( e-e

M. Karyotaki is with N.C.S.R. 'Demokritos', Institute of Informatics and Telecommunications, Telecoms Lab Net Media Lab, Agia Paraskevi, 153 10, Athens, Greece (e-mail: karyotakimaria@gmail.com)

Submitted 23 October 2013. Published as re-submitted by the authors 02 November 2013. 